\title{
Epífora secundaria a punto lagrimal supernumerario tratada con tapones de vías lagrimales. Presentación de un caso y revisión de la literatura
}

\author{
Epiphora due to a supernumerary lacrimal punctum treated with lacrimal plugs. \\ Case presentation and literature review \\ Carlos Fleitman Broder ${ }^{1,2 *}$ y Jacobo Fleitman Chazan ${ }^{2}$ \\ ${ }^{1}$ Instituto de Oftalmología, Centro Médico ABC; ${ }^{2}$ Servicio de Oftalmología, Active Vision Center. Ciudad de México, México
}

\begin{abstract}
Resumen
Antecedentes: Los puntos lagrimales supernumerarios son una malformación congénita poco común y con una variabilidad anatómica importante. En muchas ocasiones pueden ser sintomáticos y cursar con epífora. Caso clínico: Se describe el caso de un paciente de 45 años con epífora secundaria a un punto lagrimal supernumerario sin otra patología lagrimal asociada tratado exitosamente con el uso de tapones de vías lagrimales. Conclusiones: Los tapones de vías lagrimales para el tratamiento de la epífora secundaria a puntos lagrimales supernumerarios pueden ser utilizados en pacientes con variantes anatómicas adecuadas.
\end{abstract}

Palabras clave: Epifora. Punto lagrimal. Punto lagrimal supernumerario.

\begin{abstract}
Background: Supernumerary lacrimal punctum is a rare malformation of the lacrimal apparatus with a highly variable anatomical presentation. In many cases this can be symptomatic and cause tearing. Case presentation: The case of a 45 year old male with a supernumerary lacrimal punctum and tearing without any other associated lacrimal system anomaly treated successfully with lacrimal plugs. Conclusions: Lacrimal plugs can be used as an alternative treatment in selected cases of symptomatic supernumerary lacrimal punctum with epiphora.
\end{abstract}

Key words: Epiphora. Lacrimal punctum. Supernumerary lacrimal punctum.

\section{Introducción}

Los puntos lagrimales supernumerarios son una malformación congénita poco común. Su incidencia se calcula en 1/60,000 y la localización más común es a lo largo de la línea del canalículo inferior, aunque también pueden encontrarse en la carúncula o en el fondo de saco conjuntival medial'.

Generalmente son asintomáticos y su diagnóstico suele ser incidental; sin embargo, en algunos pacientes

\section{Correspondencia:}

${ }^{*}$ Carlos Fleitman-Broder

Avda. Vasco de Quiroga, 4001

Cuajimalpa

Fecha de recepción: 16-05-2019

C.P. 05370, Ciudad de México, México

E-mail: cfleitman@gmail.com
Cir Cir. 2020;88(S1):28-30

Contents available at PubMed www.cirugiaycirujanos.com

0009-7411/@ 2019 Academia Mexicana de Cirugía. Publicado por Permanyer. Este es un artículo open access bajo la licencia CC BY-NC-ND (http://creativecommons.org/licenses/by-nc-nd/4.0/). 
pueden llegar a causar epífora secundaria a otras patologías de la vía lagrimal o a reflujo de lágrimas por el punto accesorio ${ }^{1,2}$.

\section{Caso clínico}

Paciente varón de 45 años que refiere presentar epífora en el ojo derecho durante toda la vida al exponerse a condiciones de viento, lectura prolongada 0 aire acondicionado. Sin embargo, al ser solo ocasionalmente nunca le ha dado importancia, pero se ha vuelto más frecuente.

En la exploración física se encuentra un punto lagrimal supernumerario proximal al punto lagrimal normal (Fig. 1). Con el sondeo y la irrigación de los puntos lagrimales se observa que ambos tienen su propio canal lagrimal y que drenan en el saco lagrimal; así mismo, se confirma que la vía lagrimal inferior se encuentra permeable a la irrigación. El resto de la exploración oftalmológica, incluyendo la evaluación de la superficie ocular, fue completamente normal. Se inició tratamiento con lubricantes tópicos con la intención de disminuir la producción de lágrimas, con mejoría parcial.

Se decidió hacer una prueba terapéutica de forma empírica con un tapón de punto lagrimal de colágena (SOFT PLUG ${ }^{\circledR}$ Collagen, OASIS, Glendora, CA, USA) (Fig. 2) en el punto lagrimal distal, con resolución de la sintomatología por un periodo de 2 semanas y recurrencia al disolverse el tapón, por lo que se procedió a la colocación de un tapón canalicular definitivo de hidrogel (FORM FIT ${ }^{\oplus}$ Canalicular plug, OASIS, Glendora, CA, USA), con resolución completa de la sintomatología y sin recurrencia a los 3 meses de seguimiento.

\section{Discusión}

Las vías lagrimales se originan del ectodermo superficial que invagina en la zona medial de los párpados y en el surco que se forma entre los procesos frontonasal y maxilar. La teoría más aceptada de la formación de los puntos supernumerarios es que existe una separación incompleta del tejido invaginado de la superficie ectodérmica o que hay brotes anormales de la porción proximal del cordón ectodérmico ${ }^{3,4}$.

Esta condición se ha asociado a otras patologías de la vía lagrimal, como obstrucción del conducto nasolagrimal, fístulas, divertículos del saco lagrimal y agenesia monocanalicular ${ }^{5}$. En estos pacientes existe una causa clara para la epífora, pero en aquellos en

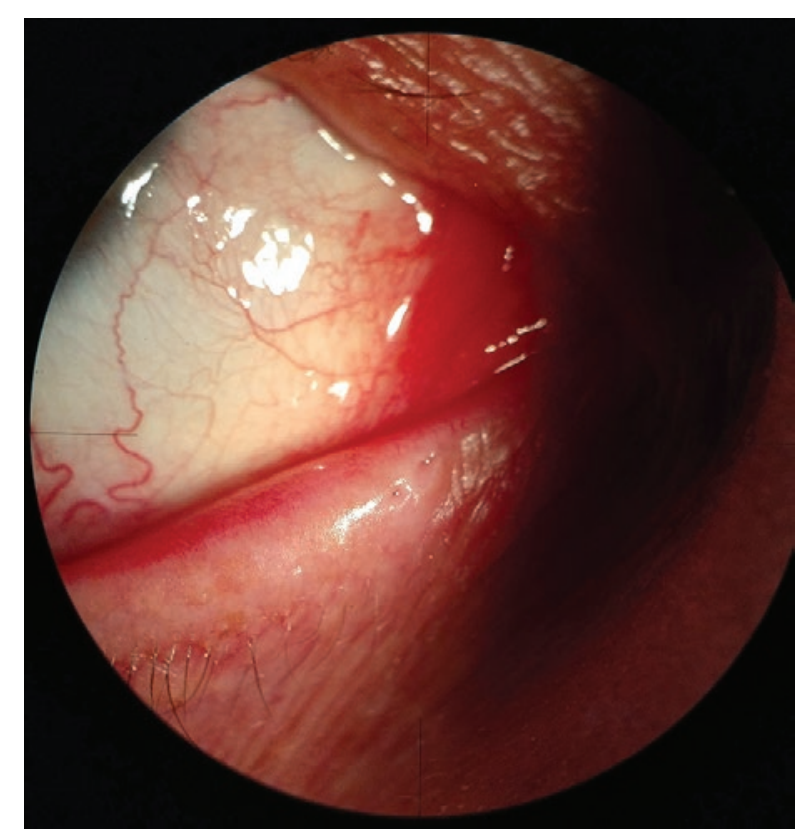

Figura 1. Punto lagrimal supernumerario en el párpado inferior del ojo derecho.

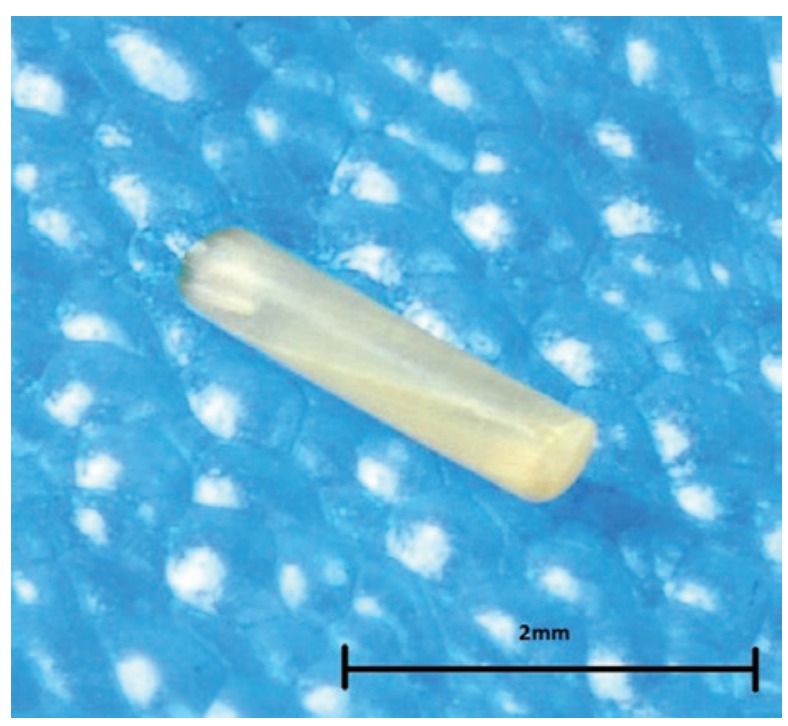

Figura 2. Tapón de puntos lagrimales de colágeno temporal.

los que no se encuentra otra anomalía asociada con la vía lagrimal el mecanismo fisiopatológico aún no ha sido dilucidado por completo.

La teoría clásica del mecanismo de bomba que ejerce el saco lagrimal para lograr un adecuado drenaje ${ }^{6}$ se ha enriquecido con la teoría basada en estudios anatómicos de cadáveres realizados por Kakizaki, et al. ${ }^{7}$, según la cual los canalículos se encuentran divididos en dos compartimentos por el músculo de 
Horner, y esta relación provoca un alargamiento de los canalículos contribuyendo al mecanismo de bombeo. En el caso de nuestro paciente, la alteración de este mecanismo de bombeo podría ser la causa de la epífora, ya sea disminuyendo la cantidad de lágrima que es capaz de drenar el sistema o provocando reflujo por el punto y el canalículo accesorios.

La prueba terapéutica con un tapón de vías lagrimales de colágeno temporal confirmó que la epífora presentada por el paciente era secundaria al punto supernumerario.

En el caso presentado, ambos puntos drenan a su propio canalículo, por lo se tomó la decisión de colocar un tapón canalicular de hidrogel para el tratamiento definitivo. Esto no podría haberse realizado si ambos puntos lagrimales compartieran un canalículo común; cuando esto ocurre es conveniente valorar el uso de tapones de silicona para punto lagrimal, lo cual presenta el inconveniente de tener una menor tasa de retención, que probablemente sea aún menor en los puntos lagrimales accesorios porque suelen tener una forma ahusada.

Hasta el día de hoy no encontramos en la literatura casos reportados del uso de tapones de vías lagrimales para el tratamiento de la epífora secundaria a un punto lagrimal supernumerario. En un inicio parecería contraintuitivo el uso de esta modalidad terapéutica en un paciente que refiere lagrimeo, pero al tomar en cuenta la alteración del mecanismo fisiológico de bombeo lagrimal causada por esta malformación congénita es posible explicar el éxito terapéutico con esta modalidad.

Otra ventaja de intentar el tratamiento con tapones de vías lagrimales es que su colocación es un procedimiento mínimamente invasivo que se realiza en el consultorio y, en caso de causar algún efecto no deseado, es $100 \%$ reversible.

Dado que esta es una afección poco común y con una alta variabilidad en cuanto a las características anatómicas que presentan los pacientes, no existe un tratamiento único para todos los casos de puntos supernumerarios. Cuando se encuentra un sistema canalicular independiente en cada uno de los puntos lagrimales es prudente valorar el tratamiento con tapones de vías lagrimales si el paciente presenta epífora.

\section{Conflicto de intereses}

Los autores declaran no tener conflictos de intereses.

\section{Responsabilidades éticas}

Protección de personas y animales. Los autores declaran que para esta investigación no se han realizado experimentos en seres humanos ni en animales.

Confidencialidad de los datos. Los autores declaran que han seguido los protocolos de su centro de trabajo sobre la publicación de datos de pacientes.

Derecho a la privacidad y consentimiento informado. Los autores han obtenido el consentimiento informado de los pacientes y/o sujetos referidos en el artículo. Este documento obra en poder del autor de correspondencia.

\section{Bibliografía}

1. Ali MJ. Supernumerary puncta. En: Atlas of lacrimal drainage disorders. Singapore: Springer; 2015, pp. 201-203.

2. Kirk RC. Developmental anomalies of the lacrimal passages. A review of the literature and presentation of three unusual cases. Am J Ophthalmol. 1956;42:227-32.

3. Sevel D. Development and congenital abnormalities of the nasolacrimal apparatus. J Pediatr Ophthalmol Strabismus. 1981;18:13-9.

4. Ali MJ. Embryology of the lacrimal drainage system. En: Atlas of lacrimal drainage disorders. Singapore: Springer; 2015, pp. 1-6.

5. Satchi K, McNab AA. Double lacrimal puncta: clinical presentation and potential mechanisms of epiphora. Ophthalmology. 2010;117:180-3.

6. Kakizaki H, Ali MJ. Anatomy, physiology, and immunology of the lacrimal system. En: Ali M, editor. Principles and practice of lacrimal surgery. Singapore: Springer; 2018, pp-19-39.

7. Kakizaki H, Zako M, Miyaishi O, Nakano T, Asamoto K, Iwaki M. The lacrimal canaliculus and sac bordered by the Horner's muscle form the functional lacrimal drainage system. Ophthalmology. 2005;112:710-6. 